\title{
WEAK EIGENVECTORS AND THE FUNCTIONAL CALCULUS
}

\author{
BY \\ R. T. HARRIS(1)
}

In this paper we are concerned with the problem of constructing the functional calculus for an operator with continuous spectrum from a given collection of weak eigenvectors. A functional calculus is a homomorphism $f \rightarrow T_{f}$ of an algebra of functions defined over the 'spectrum' $V$ into a subalgebra of bounded linear operators acting in a banach space $E$; we may regard an 'eigenfunction expansion' for such a functional calculus as an expression for all $T_{f}$ over a banach space $D$ dense in $E$ of the form $T_{f}=\int_{V} f(\lambda) T_{\lambda} d m(\lambda)$, where $m$ is a measure over $V$ and $T_{\lambda}$ is a bounded linear operator from $D$ into the space of 'distributions' $D^{\prime}$ '. In general, it is hoped that the range of $T_{\lambda}, \operatorname{Im} T_{\lambda}$, will consist of rather precisely specified weak eigenvectors (specified, for example, by boundary values). Since a family of weak eigenvectors is often available, a problem that often appears in applications is the following: Given a family of weak eigenvectors at $\lambda$, when is it true that this family coincides with $\operatorname{Im} T_{\lambda}$ ?

There are many results in the literature for a closed operator with dense domain in a banach space with continuous spectrum which guarantee a rich functional calculus and a representation of the functional calculus in terms of an eigenfunction expansion where, in fact, $\operatorname{Im} T_{\lambda}$ consists of weak eigenvectors. F. Browder [3] established such results for a large class of nonsymmetric partial differential operators, and Gel'fand and Šilov [5] were the first to consider the problem of the existence of eigenfunction expansions in general operator theoretic terms. However there are few results that tell us which weak eigenvectors to choose for the eigenfunction expansion. Most results of this nature are confined to differential operators with strong regularity theorems, the most classical being Weyl's construction of the Plancherel formula for a singular second order ordinary differential operator [10]. The crucial step is the 'Weyl lemma' which asserts that certain weak eigenvectors are actually infinitely differentiable, and so, classical uniqueness theorems may be invoked to determine precisely which weak eigenvectors contribute to the eigenfunction expansion.

The results given here, in contrast, are well within the context of functional

Received by the editors September 7, 1962.

(1) This research was supported by the United States Air Force through the Air Force Office of Scientific Research of the Office of Aerospace Research under Contract AF 49638892 and Grant Af AFOSR 61-51. 
analysis and so, in some respects, afford more generality. Other than the traditional machinery of functional analysis and harmonic analysis, the results in this paper depend decisively upon certain results of A. Beurling which are stated in $\$ 1$. For our purposes, these results essentially establish a usable bridge between the resolvent and its boundary values.

The methods used in this paper require, ab initio, that we restrict our consideration to closed operators with dense domain in a banach space which generate a one-parameter strongly continuous group of operators in this banach space. In view of the fact that the Hille-Yoshida theorem (cf. Theorem 12.3.2 [6]) gives necessary and sufficient conditions on the resolvent in order that the operator generate such a group of operators and the various exponential formulas can relate conditions on the resolvent to conditions on the group of operators, we formulate our results directly in terms of a one-parameter strongly continuous group of operators in a banach space. A certain amount of complexity in stating our results is avoided in this way and (hopefully) this will not mitigate their usefulness.

$\$ 1$ presents the various classes of one-parameter groups of operators for which we have results, and gives the essential definitions and technical apparatus that will be needed. $\$ 2$ constructs the functional calculus and the generalized eigenfunction expansion; it is also shown that under relatively mild circumstances, the functional calculus is particularly rich in operators which approximate spectral projections over a compact set. $\S 3$ is the body of the paper; here, the relation between the functional calculus and weak eigenvectors is explored in some detail. Our principal results are Theorems 2 and 5, which give explicit criteria for the selection of the weak eigenvectors that appear in the functional calculus. In $\S 4$, by way of an application, we show how a unitary equivalence may, under suitable circumstances, be given a representation by means of weak eigenvectors.

1. Definitions and preliminaries. By $M_{b}$, we denote the usual space of bounded complex Radon measures over the real line and by $L^{1}$, the closed subspace of $M_{b}$ consisting of those bounded measures which are absolutely continuous with respect to lebesgue measure. As usual, $m \in L^{1}$ will be labeled by its derivative, and any function which is equal almost everywhere to such a function will be called integrable. We make $M_{b}$ into a banach algebra by defining the convolution product for $m, n \in M_{b}: m^{*} n(f)=\iint f(x+t) d m(t) d n(x), f$ continuous with compact support. $L^{1}$ becomes a closed subalgebra of $M_{b}$. We will denote the fourier transform of $m \in M_{b}, \int e^{i t \lambda} d m(\lambda)$, by $\hat{m}(t)$.

All linear spaces considered in this paper will be linear spaces over the field of complex numbers.

Let $D$ and $E$ be fixed separable banach spaces with conjugate duals $D^{\prime}$ and $E^{\prime}$ for which $D \subseteq E \subseteq E^{\prime} \subseteq D^{\prime}$, where all maps induced by inclusion are assumed continuous with dense range. In addition, we require that the duality between $D^{\prime}$ 
and $D$ should coincide with the duality between $E^{\prime}$ and $E$ when restricted to $E^{\prime}$ and $D$, with this requirement, we may denote without confusion the result of applying an element $x^{\prime} \in D^{\prime}$ (or $E^{\prime}$ ) to an element $x \in D$ (or $E$ ) by $\left\langle x, x^{\prime}\right\rangle . D^{\prime}$ and $E^{\prime}$ may be regarded as playing the roles of spaces of distributions.

We denote by $\mathscr{R}$ the collection of all strongly continuous representations of the additive group of the real line into a subgroup of $L(E)$, the usual algebra of bounded operators of $E$ into $E$, where the group composition in $L(E)$ is the composition of operators. If $r \in \mathscr{R}$, then the value of $r$ at a real number $t$ will be denoted by $r(t)$. We make no assumptions of boundedness for the representations in $\mathscr{R}$.

Associated with each $r \in \mathscr{R}$ is the real-valued function $p_{r}$ given by $p_{r}(t)=\left|{ }^{*} r(t)\right|_{L\left(D, D^{\prime}\right)}$ the norm of the adjoint of $r(t)$ considered as an operator from $D$ into $D^{\prime}$; since $p_{r}$ is lower semi-continuous, $p_{r}$ is lebesgue measurable.

Definition. $\mathscr{L}$ will denote those $r \in \mathscr{R}$ which satisfy

(1) $p_{r}$ is integrable.

(2) For each $a, b \in D$, the fourier transform of the function $t \rightarrow\langle r(t) a, b\rangle$ is integrable.

If $r$ is generated by a spectral operator of scalar type with condition (1) satisfied, then (2) is automatically satisfied. A substantially large class of operators with continuous spectrum encountered in applications generate $r \in \mathscr{L}$. We will write $\rho_{a, b}$ for the fourier transform of the function $t \rightarrow\langle r(t) a, b\rangle$.

Definition. $\mathscr{L}_{1}$ will denote those $r \in \mathscr{L}$ for which $t \rightarrow|r(t)|_{L(E)}$ is continuous and the integral $\int\left(\log ^{+}|r(t)| /\left(1+t^{2}\right)\right) d t$ is finite.

The function $w_{r}(t)=\sup \left(|r(t)|_{L(E)},|r(-t)|_{L(E)}, 1\right)$ for $r \in \mathscr{R}$ is a measurable, even, submultiplicative function for which $w_{r}(t) \geqq w_{r}(0)=1$, for all $t$. We let $S(r)$ denote the algebra under pointwise multiplication of functions which are fourier transforms of those $m \in M_{b}$ for which $\int w_{r} d|m|<+\infty$, and, by $A(r)$, we denote the subalgebra of $S(r)$ consisting of the fourier transforms of functions in $L^{1}$. If $f \in S(r), m_{f}$ will denote the measure such that $\hat{m}_{f}=f$.

Should $r \in \mathscr{L}_{1}$, then $w_{r}$ is continuous and satisfies $\int\left(\log w_{r}(t) /\left(1+t^{2}\right)\right) d t$ $<+\infty$. Under these circumstances, a result of A. Beurling gives us the following theorem for $A(r)$ (essentially Theorem II.B [1, p. 356] and [2]—for the terminology concerning banach algebras, cf. [7]).

Theorem (Beurling). Let $r \in \mathscr{L}_{1} ;$ give $A(r)$ the norm $|f|_{r}=\int w_{r} d\left|m_{f}\right|$ and complex conjugation as an involution. Then $A(r)$ becomes a commutative semisimple regular $B^{*}$-algebra. Let $I$ be a compact interval on the line and let $\mathscr{I}_{I}$ be the closed *-ideal in $A(r)$ given by the set of $f \in A(r)$ vanishing over $I$. Form the quotient $B^{*}$-algebra $A(r)_{I}$ by the ideal $\mathscr{I}_{I}$ and denote the norm in this algebra by ||$_{r, I}$. Then, for each $\tilde{f}$, f denoting the image in $A(r)_{I}$ by $f \in A(r)$, the spectral norm of $\tilde{f}$ is given by

$$
\lim _{n \rightarrow \infty}\left|f^{n}\right|_{r, I}^{1 / n}=\sup _{\lambda \in I}|f(\lambda)|
$$


As a consequence of this result, the *-isomorphism defined by mapping $f$ into the restriction of $f$ to $I$ is the Gel'fand representation of $A(r)_{I}$. Since $A(r)$ is a regular $B^{*}$-algebra, $A(r)_{I}$ has a unit. Hence, by the well-known analogue of the Wiener lemma for commutative $B^{*}$-algebras with unit $[7, \mathrm{p} .71], f^{-1}$ exists in $A(r)_{I}$ if and only if $f$ does not vanish over $I$. That is to say, there exists a $g \in A(r)$ such that $g=1 / f$ over $I$ if and only if $f$ does not vanish over $I$.

The last important type of $r \in \mathscr{R}$ for which we have results is the following generalization of the unitary representation.

DEFINITION. $\quad r \in \mathscr{R}$ will be called subunitary if

(1) For each nonzero $x \in E,\langle x, x\rangle$ is real and strictly positive.

(2) For all $t$, the restriction of ${ }^{*} r(t)$ to $E$ coincides with $r(-t)$.

As is well known, the fact that $\langle x, x\rangle$ is real for all $x \in E$ implies that the sesquilinear form over $E, x, y \rightarrow\langle x, y\rangle$ is hermitian.

Finally, for a given $r$ under consideration and a complex number $\lambda$, we let $\mathscr{E}_{\lambda}$ denote the subspace of $E^{\prime}$ of $e \in E^{\prime}$ for which ${ }^{*} r(t) e=e^{i \lambda t} e$ for all $t$. Clearly $e \in \mathscr{E}_{\lambda}$ if and only if, for all $t, x \in E,\langle r(t) x, e\rangle=e^{i \lambda t}\langle x, e\rangle$. More generally, for any linear space of vectors $N$ dense in $D$ for which $r(t)$ maps $N$ into $N$ for each $t$, we denote by $\mathscr{N}_{\lambda}$ the subspace of $D^{\prime}$ of $e \in D^{\prime}$ for which $\langle r(t) a, e\rangle=e^{i \lambda t}\langle a, e\rangle$ for all $t$ and all $a \in N$. If $\mathscr{E}_{\lambda} \neq\{0\}\left(\mathscr{N}_{\lambda} \neq\{0\}\right)$, then the nonzero elements of $\mathscr{E}_{\lambda}\left(\mathscr{N}_{\lambda}\right)$ is the set of weak eigenvectors in $D^{\prime}$ over $E$ (over $N$ ). We will let $S$ denote the set of $\lambda, \lambda$ an arbitrary complex number, such that $\mathscr{E}_{\lambda} \neq\{0\}$.

2. The functional calculus and the eigenfunction expansion. Following a procedure originating with R. S. Phillips for semi-groups [8], we construct a spectral calculus for $r \in \mathscr{R}$.

Proposition 1. Let $r \in \mathscr{R}$. For each $f \in S(r)$.

$$
T_{f} x=\int r(t) x d m_{f}(t)
$$

exists and defines an operator in $L(E)$ which commutes with $r(t)$ for all $t$. The map defined by $f \rightarrow T_{f}$ is an algebraic homomorphism onto a subalgebra of $L(E)$ for which $\left|T_{f}\right|_{L(E)} \leqq|f|_{r}$.

Proof. Since $\int r(t) x d m_{f}(t)$ exists as an ordinary vector-valued Riemann integral, we have

$$
r\left(t^{\prime}\right) \int r(t) x d m_{f}(t)=\int r(t) r\left(t^{\prime}\right) x d m_{f}(t)
$$

so that $r\left(t^{\prime}\right) T_{f} x=T_{f} r\left(t^{\prime}\right) x$, for all $x \in E$.

An application of the Fubini theorem gives

$$
T_{f} T_{g} x=\int r(t) x d\left(m_{g}{ }^{*} d m_{f}\right)(t)=\int r(t) x d m_{f g}(t)=T_{f g} x
$$


so that the fact that $f \rightarrow T_{f}$ is an algebraic homomorphism onto a subalgebra of $L(E)$ follows from the fact that $S(r)$ is an algebra. Clearly

$$
\left|T_{f}\right|_{L(E)} \leqq \int w_{r}(t) d\left|m_{f}\right|(t) \equiv|f|_{r}
$$

Proposition 2. Let $r \in \mathscr{L}$. Then, there exists a unique map from the real line into $L\left(D, D^{\prime}\right), \lambda \rightarrow T_{\lambda}$, such that for each pair $a, b \in D, \lambda \rightarrow\left\langle a, T_{\lambda} b\right\rangle$ is $a$ continuous integrable function with

$$
\int f\left\langle a, T_{\lambda} b\right\rangle d \lambda=\left\langle T_{f} a, b\right\rangle, \quad f \in S(r)
$$

and $\left|T_{\lambda}\right|_{L\left(D, D^{\prime}\right)} \leqq \int p_{r}(t) d t$ for all $\lambda$.

Proof. Uniqueness is a consequence of the fact that any equivalence class of functions in $L^{1}$ contains at most one continuous function. As

$$
\left|\rho_{a, b}(\lambda)\right| \leqq \int|\langle r(t) a, b\rangle| d t \leqq|a|_{D}|b|_{D} \int p_{r}(t) d t
$$

we have for each $b \in D$, a unique $T_{\lambda} b \in D^{\prime}$ for which $\left\langle a, T_{\lambda} b\right\rangle=\rho_{a, b}(\lambda)$. Since the map $a, b \rightarrow \rho_{a, b}(\lambda)$ is sesquilinear, it is evident that $T_{\lambda}$ is a linear map from $D$ into $D^{\prime}$; as $\left|\left\langle a, T_{\lambda} b\right\rangle\right| \leqq|a|_{D}|b|_{D} \int p_{r}(t) d t$, we have $\left|T_{\lambda}\right|_{L\left(D, D^{\prime}\right)} \leqq \int p_{r}(t) d t$. Now, an application of the Fubini theorem yields:

$$
\begin{aligned}
\left\langle T_{f} a, b\right\rangle & =\int\langle r(t) a, b\rangle d m_{f}(t)=\int d m_{f}(t) \int e^{i \lambda t}\left\langle a, T_{\lambda} b\right\rangle d \lambda \\
& =\int\left\langle a, T_{\lambda} b\right\rangle d \lambda \int e^{i \lambda t} d m_{f}(t)=\int f\left\langle a, T_{\lambda} b\right\rangle d \lambda .
\end{aligned}
$$

For $r \in \mathscr{L}$, we let $V_{D}$ denote the set of all $\lambda$ such that $T_{\lambda} \neq 0 ; V_{D}$ is an open subset of the real line, since $V_{D}=\bigcup_{a, b \in D}\left\{\lambda: \rho_{a, b}(\lambda) \neq 0\right\}$. Proposition 2 is a rarefied version, for $r \in \mathscr{L}$, of the classical idea of an eigenfunction expansion and $V_{D}$ plays the role of that part of the spectrum of $r$ which contributes to the eigenfunction expansion.

Proposition 3. Let $r \in \mathscr{R}$. Then, for all $f \in S(r), e \in \mathscr{E}_{\lambda},{ }^{*} T_{f} e=f(\lambda) e$.

Proof. Let $x \in E$; then

$$
\left\langle x,{ }^{*} T_{f} e\right\rangle=\left\langle T_{f} x, e\right\rangle=\int\langle r(t) x, e\rangle d m_{f}(t)=\int e^{i \lambda t}\langle x, e\rangle d m_{f}(t)=\langle x, e\rangle f(\lambda) .
$$

Since this is true for all $x \in E$, we have ${ }^{*} T_{f} e=f(\lambda) e$.

Denote by $S\left(r, V_{D}\right)$ the functions defined over $V_{D}$ which are the restriction of functions in $S(r)$. For $r \in \mathscr{L}_{1}$, we have the following improvement of Proposition 1.

Proposition 4. If $r \in \mathscr{L}_{1}$, then the map $f \rightarrow T_{f}$ considered as a map from $S\left(r, V_{D}\right)$ into $L(E)$ is an algebraic isomorphism for which 


$$
\left|T_{f}\right|_{L\left(D, D^{\prime}\right)} \leqq|f|_{L^{1}} \int p_{r}(t) d t .
$$

Proof. Let $f$ be such that $T_{f}=0$. Then, for all $g \in S(r), 0=T_{f} T_{g}=T_{f g}$, so that for all $a, b$ in $D$,

$$
\int f g \rho_{a, b} d \lambda=0
$$

Assuming that $f$ is not 0 over $V_{D}$ implies that there is a real number $\lambda_{0}$ and some $a, b \in D$ such that $\operatorname{Re}\left\{f\left(\lambda_{0}\right) \rho_{a, b}\left(\lambda_{0}\right)\right\}>0$. Since $f$ and $\rho_{a, b}$ are continuous, there exist an open nonvoid interval $I \subseteq V_{D}$ such that for all $\lambda \in I, \operatorname{Re}\left\{f(\lambda) \rho_{a, b}(\lambda)\right\}>0$. Since $A(r)$ is a regular $B^{*}$-algebra, there exists a real-valued strictly positive function $g_{I} \in A(r)$ whose support lies entirely within $I$. Hence,

$$
\left|\int f g_{I} \rho_{a, b} d \lambda\right| \geqq \operatorname{Re} \int f g_{I} \rho_{a, b} d \lambda=\int \operatorname{Re}\left(f g_{I} \rho_{a, b}\right) d \lambda
$$

and since the integrand is strictly greater than zero over a set of positive measure, this last integral must be strictly greater than zero. This is a contradiction, so that $f(\lambda)=0$ for all $\lambda \in V_{D}$. Finally,

$$
\left|T_{f}\right|_{L\left(D, D^{\prime}\right)} \leqq \int|f|\left|T_{\lambda}\right|_{L\left(D, D^{\prime}\right)} d \lambda \leqq|f|_{L^{1}} \int p_{r}(t) d t
$$

by Proposition 2.

As a consequence of the above, we have the following result which expresses a striking similarity between the functional calculus of $r \in \mathscr{L}_{1}$ and the functional calculus for a spectral operator of scalar type. Let $\mathscr{V}$ denote the set of pairs of subsets of $V_{D},(A, B), A \subseteq B$, where in $V_{D}, A$ and $B$ are nonvoid and open with $\bar{B}$ compact.

THEOREM 1. If $r \in \mathscr{L}_{1}$, then for each $(A, B) \in \mathscr{V}$, there exists a nonzero $p(A, B) \in L(E)$ which commutes with $r(t)$ for all $t$ and satisfies the following properties:

(a) For $(A, B), \quad\left(A^{\prime}, B^{\prime}\right) \in \mathscr{V}, \quad p(A, B) p\left(A^{\prime}, B^{\prime}\right)=p\left(A^{\prime}, B^{\prime}\right) p(A, B) \quad$ and $\quad$ if $B^{\prime} \cap B=\varnothing$, then $p(A, B) p\left(A^{\prime}, B^{\prime}\right)=0$.

(b) If $F_{A}$ denotes the filter of open sets containing $A$, then

$$
\lim _{B \in F_{A}}\left|p(A, B)^{2}-p(A, B)\right|_{L\left(D, D^{\prime}\right)}=0 .
$$

(c) If $\left\{\left(A_{n}, B_{n}\right)\right\}$ is a sequence in $\mathscr{V}$, such that $\left\{A_{n}\right\}$ is a rising sequence whose union is $V_{D}$, then for $a, b \in D, \lim _{n}\left\langle p\left(A_{n}, B_{n}\right) a, b\right\rangle=\langle a, b\rangle$.

Proof. Using, once more, the fact that the $B^{*}$-algebra $A(r)$ is regular, for any $(A, B) \in \mathscr{V}$, there is a real positive $f_{A, B} \in A(r)$ which vanishes outside of $B$ and is identically 1 over $A$; we may also assume that the range of $f_{A, B}$ lies in the interval $[0,1]$. Since $f_{A, B} \neq 0$ over $V_{D}$, Proposition 4 implies that $T_{f_{A, B}} \neq 0$. We set $T_{f_{A, B}}=p(A, B)$. 
Proposition 1 immediately implies (a) and the fact that $p(A, B)$ commutes with $r(t)$ for all $t$. Using the estimate in Proposition 4, we have

$$
\begin{aligned}
\left|p(A, B)^{2}-p(A, B)\right|_{L\left(D, D^{\prime}\right)} & \leqq\left\{\int\left|\left(f_{A, B}^{2}-f_{A, B}\right)\right| d \lambda\right\} \int p_{r}(t) d t \\
& \leqq\left\{\int_{B \cap \mathscr{C} A} d \lambda\right\} \int p_{r}(t) d t
\end{aligned}
$$

and since $\inf _{B \in F_{A}} \int_{B \cap \mathscr{C} A} d \lambda=0$, this proves (b). Finally, $\lim _{n}\left\langle p\left(A_{n}, B_{n}\right) a, b\right\rangle$ $=\lim _{n} \int f_{A_{n}, B_{n}} \rho_{a, b} d \lambda=\int \rho_{a, b} d \lambda=\langle a, b\rangle$, using the Lebesgue dominated convergence theorem.

Proposition 5. For each $a$ in the unit ball of $D$, there exists a decomposition $a=x_{1}+x_{2}$ such that $x_{1}, x_{2}$ are in the images in $E$ of the unit ball of $D$ by the operators $p(A, B)$ and $1-p(A, B)$, respectively. If $x_{1}^{\prime}, x_{2}^{\prime}$ are another pair of vectors satisfying the above conditions, then

$$
\left|x_{1}-x_{1}^{\prime}\right|_{D^{\prime}}=\left|x_{2}-x_{2}^{\prime}\right|_{D^{\prime}} \leqq 4\left|p(A, B)^{2}-p(A, B)\right|_{L\left(D, D^{\prime}\right)} .
$$

Proof. The existence of such a decomposition is clear. Since $x=x_{1}-x_{1}^{\prime}$ $=x_{2}^{\prime}-x_{2}$ is a vector in the image of the ball of radius 2 in $D$ of the operation $p(A, B)$ and $1-p(A, B)$, there exists $b, c$ in the ball of radius 2 in $D$ such that $x=p(A, B) b=(1-p(A, B)) c$. Hence, $x=\left(p(A, B)-p(A, B)^{2}\right)(b+c)$, or $|x|_{D^{\prime}} \leqq 4\left|p(A, B)^{2}-p(A, B)\right|_{L\left(D, D^{\prime}\right)}$.

For $(A, B) \in \mathscr{V}$, we will denote by $p(A, B)$ any operator in $L(E)$ constructed in the manner of Theorem 1 , i.e., from a real positive function in $S(r)$ equal to 1 over $A$ and vanishing outside of $B$.

\section{The main theorems.}

Proposition 6. If $r \in \mathscr{L}_{1}$ and $N$ is a dense linear space in $D$ invariant under $r(t)$ for all $t$, then $\operatorname{Im} T_{\lambda} \subseteq \mathscr{N}_{\lambda}$ for all $\lambda \in V_{D}$.

Proof. For each $f \in A(r), \quad a \in N, \quad b \in D, \quad \int f e^{i \lambda t}\left\langle a, T_{\lambda} b\right\rangle d \lambda=\left\langle T_{f} r(t) a, b\right\rangle$ $=\int f\left\langle r(t) a, T_{\lambda} b\right\rangle d \lambda$. Since $A(r)$ is regular, $A(r)$ is dense in the space of continuous functions vanishing at infinity, in virtue of the Stone-Weierstrass theorem; hence, $e^{i \lambda t}\left\langle a, T_{\lambda} b\right\rangle=\left\langle r(t) a, T_{\lambda} b\right\rangle$ for almost all $\lambda$; as the right and left sides of this equality are continuous functions of $\lambda$, this equality holds for all $\lambda$. Hence, $\operatorname{Im} T_{\lambda} \subseteq \mathscr{N}_{\lambda}$ for all $\lambda \in V_{D}$.

For $r \in \mathscr{L}_{1}$, we have the inclusions $\mathscr{E}_{\lambda} \subseteq \mathscr{N}_{\lambda}$ and $\operatorname{Im} T_{\lambda} \subseteq \mathscr{N}_{\lambda}$; these inclusionswithout 'regularity'-will, in general, be proper. The question we now consider is that of finding reasonable conditions under which we can assert that $\mathscr{E}_{\lambda}=\operatorname{Im} T_{\lambda}$.

Let $V_{D}(c)$ denote the open subset of $V_{D}$ consisting of those $\lambda$ such that there exists $a \in D$ for which $\rho_{a, c}(\lambda) \neq 0$.

Proposition 7. If $r \in \mathscr{L}_{1}$ and there exists $c \in D$ such that the linear span of $\left\{{ }^{*} r(t) c\right\}_{t}$ is dense in $E^{\prime}$, then $V_{D}(c)$ is dense in $V_{D}$. 
Proof. If $V_{D}(c)$ is not dense, then there exists $(A, B) \in \mathscr{V}$ such that $B \cap \overline{V_{D}(c)}=\emptyset$. So, $\langle r(t) p(A, B) a, c\rangle=\int e^{i \lambda t} f_{A, B} \rho_{a, c} d \lambda$, and as the support of $\rho_{a, c}$ is contained in $\overline{V_{D}(c)}$, it follows that $\left\langle p(A, B) a,{ }^{*} r(t) c\right\rangle=0$, for all $t$, and so $p(A, B)=0$, which contradicts Theorem 1 .

THEOREM 2. Let $r \in \mathscr{L}_{1}$ and satisfy:

(a) $\int p_{r}(t) w_{r}(t) d t<+\infty$.

(b) There exists a $c \in D$ such that the linear span of the set of vectors $\left\{{ }^{*} r(t) c\right\}_{t}$ is dense in $E^{\prime}$. Then, for $\lambda \in S \cap V_{D}(c), \mathscr{E}_{\lambda}$ is the one-dimensional space spanned by $T_{\lambda} c$.

Proof. In virtue of the fact that $\mathscr{E}_{\lambda} \neq 0$, to show that $\mathscr{E}_{\lambda}$ is the linear span of $T_{\lambda} c$ is equivalent to showing that the hyperplane $X$ in $D\left\{a:\left\langle a, T_{\lambda} c\right\rangle=0\right\}$ $=X \subseteq\left\{a:\langle a, e\rangle=0\right.$ for all $\left.e \in \mathscr{E}_{\lambda}\right\}$. Let $a \in X$. Since $\lambda \in V_{D}(c)$, there exists $a_{0} \in D$ such that $\rho_{a_{0}, c}(\lambda) \neq 0$, so that there is a compact nonvoid interval $I$ containing $\lambda$ in its interior such that $\rho_{a_{0}, c} \neq 0$ over $I$.

Now, (a) implies that $\rho_{a, b}$ is in $A(r)$ for all $a, b \in D$, so that the aforementioned result of Beurling asserts the existence of an $f \in A(r)$ such that

$$
f=\frac{\rho_{a, c}}{\rho_{a_{0}, c}} \quad \text { over } I .
$$

Let $g \in A(r)$ where $g(\lambda)=1$ and $g$ vanishes outside of $I$. Rewriting $\left(^{*}\right)$, we have $f g \rho_{a_{0}, c}=g \rho_{a, c}$ everywhere and it follows that $\left\langle r(t) T_{f g} a_{0}, c\right\rangle=\left\langle r(t) T_{g} a, c\right\rangle$, or $\left\langle T_{f g} a_{0},{ }^{*} r(t) c\right\rangle=\left\langle T_{g} a,{ }^{*} r(t) c\right\rangle$. From this expression and condition (b), we have

$$
T_{f g} a_{0}=T_{g} a .
$$

Let $e$ be an arbitrary element in $\mathscr{E}_{\lambda}$. Using Proposition 3 and $(* *)$, we have

$$
\langle a, e\rangle=g(\lambda)\langle a, e\rangle=\left\langle T_{g} a, e\right\rangle=\left\langle T_{f g} a_{0}, e\right\rangle=f(\lambda) g(\lambda)\left\langle a_{0}, e\right\rangle
$$

from $\left(^{*}\right), f(\lambda)=0$, so that $\langle a, e\rangle=0$. That is to say $X \subseteq\{a:\langle a, e\rangle=0$, for all $\left.e \in \mathscr{E}_{\lambda}\right\}$, which is what was to be proved.

Theorem 2 is essentially a uniqueness theorem for weak eigenvectors in $E^{\prime}$. The following two corollaries emphasize this aspect of the theorem.

COROLLARY 1. If $r$ and $c$ satisfy the conditions of Theorem 2, then for any $\lambda \in V_{D}(c)$ such that $\operatorname{Im} T_{\lambda} \subseteq \mathscr{E}_{\lambda}, \operatorname{Im} T_{\lambda}=\mathscr{E}_{\lambda}$ and both of these spaces are onedimensional.

Proof. The fact that $\lambda \in V_{D}(c)$ implies that $T_{\lambda} \neq 0$, so that $\mathscr{E}_{\lambda} \neq\{0\}$; hence, Theorem 2 is applicable.

COROLlaRY 2. Let $r$ and $c$ satisfy the conditions of Theorem 2 and assume that $N$ is a linear space dense in $D$ and left invariant by $r(t)$, for all $t$. Then, given any $\lambda \in V_{D}($ c $)$ for which $\mathscr{N}_{\lambda} \subseteq E^{\prime}$, we must have: $\mathscr{N}_{\lambda}=\operatorname{Im} T_{\lambda}=\mathscr{E}_{\lambda}$, and each of these spaces is one-dimensional. 
Proof. Since $\mathscr{N}_{\lambda} \subseteq E^{\prime}, \mathscr{E}_{\lambda}=\mathscr{N}_{\lambda}$, so that $\mathscr{E}_{\lambda}=\mathscr{N}_{\lambda} \supseteq \operatorname{Im} T_{\lambda}$; from this, Corollary 1 gives the result.

We now give a prescription for the construction of the functional calculus from weak eigenvectors.

COROLlary 3. Assume that $r$ and $c$ satisfy the conditions of Theorem 2 and in addition, $S \supseteq V_{D}(c)$ and $\operatorname{Im} T_{\lambda}$ is one-dimensional for all $\lambda \in V_{D}(c)$. Then there exists a pair of continuous functions $\lambda \rightarrow e_{\lambda}$ and $\lambda \rightarrow f_{\lambda}$ from $V_{D}(c)$ into $D^{\prime}, D^{\prime}$ given the weak* topology, such that $e_{\lambda}$ is a nonzero element of $\mathscr{E}_{\lambda}$ and for all $f \in S(r)$ whose support lies in $V_{D}(\mathrm{c})$,

$$
\left\langle T_{f} a, b\right\rangle=\int f\left\langle a, e_{\lambda}\right\rangle\left\langle\overline{b, f_{\lambda}}\right\rangle d \lambda
$$

Proof. Let $e_{\lambda}=T_{\lambda} c$. Since $\operatorname{Im} T_{\lambda}$ is one-dimensional for $\lambda \in V_{D}(c)$, there exists a unique $f_{\lambda} \in D^{\prime}$ such that $\left\langle a, T_{\lambda} b\right\rangle=\left\langle a, e_{\lambda}\right\rangle\left\langle\overline{b, f_{\lambda}}\right\rangle$ for all $a, b \in D$ and $\lambda \in V_{D}(c)$. By construction, the functions $\lambda \rightarrow\left\langle a, e_{\lambda}\right\rangle$ and $\lambda \rightarrow\left\langle\overline{b, f_{\lambda}}\right\rangle$ are continuous; Corollary 2 gives us the fact that $e_{\lambda} \in \mathscr{E}_{\lambda}$ and Proposition 2 gives us (*).

Without restrictions on ${ }^{*} r$ more precise information, (e.g., concerning the role of $f_{\lambda}$ ) does not seem obtainable in the present context. However, with the additional restriction that $r$ be subunitary, we can obtain a fairly classical picture of the relation between the functional calculus and the weak eigenvectors. We now restrict our attention to $r \in \mathscr{R}$ which are subunitary.

The requirement that $r$ be subunitary implies that the sesquilinear form $x, y \rightarrow\langle x, y\rangle$ is a positive definitehermitian form over $E$; that is to say, $E$ is a dense subspace of a hilbert space $H$ that one obtains by completing the prehilbert space $E$ with inner product $x, y \rightarrow\langle x, y\rangle$. Now, $\langle r(t) x, r(t) y\rangle$ $=\left\langle x,{ }^{*} r(t) r(t) y\right\rangle=\langle x, r(-t) r(t) y\rangle=\langle x, y\rangle$, so that $r(t)$ in $E$ preserves the inner product of $H$. Hence, there exists a unique unitary operator $R(t) \in L(H)$ whose restriction to $L(E)$ is $r(t)$. It is clear that $t \rightarrow R(t)$ is a unitary representation of the real line into $L(H)$, and the Banach-Steinhaus theorem assures us that $t \rightarrow R(t)$ is strongly continuous. From this, we have the following characterization of subunitary $r \in \mathscr{R}$ :

PROPOSITION 8. $\quad r$ is subunitary if and only if there exists a hilbert space $H$ and a weakly continuous unitary representation $R$ of the real line satisfying:

(i) $E$ is a dense subspace of $H$ and the inner product of $H$ coincides with $\langle$,$\rangle over E \times E$.

(ii) $R(t)$, the value of $R$ at $t$, maps $E$ continuously into $E$, and the restriction of $R(t)$ to $L(E)$ is a strongly continuous representation of the line in $L(E)$.

The natural map of $E$ into $H$ is continuous, for if $k$ is the norm of the natural map of $E$ into $E^{\prime}$, then $|x|_{H}^{2}=\langle x, x\rangle \leqq|x|_{E}|x|_{E^{\prime}} \leqq k|x|_{E}^{2}$. 
The verification of whether or not $r$ is in $\mathscr{L}$ is comparatively easy.

Proposition 9. Let $r \in \mathscr{R}$ be subunitary. Then $r \in \mathscr{L}$ if and only if $p_{r}$ is integrable.

Proof. Since $r$ is subunitary, the function $t \rightarrow\langle r(t) a, a\rangle$ for each $a \in D$ is positive definite. If $p_{r}$ is integrable, the Bochner theorem implies that its fourier transform $\rho_{a, a}$ is integrable and polarization then demonstrates that $\rho_{a, b}$ is also integrable for all $a, b \in D$; that is, $r \in \mathscr{L}$. Conversely, by definition of $\mathscr{L}, p_{r}$ is integrable.

As is well known, $R$ has an infinitesimal generator $i A$, where $A$ is a closed selfadjoint operator with dense domain in $H$. Let $V$ denote the closed subset of the real line which is the spectrum of $A$. Now, the spectral theorem of M. H. Stone (Theorem 22.4.3 [6]) yields the existence of a family of measures $m_{x, y} \in M_{b}$ for all $x, y \in H$ such that $\langle R(t) x, y\rangle=\int e^{i \lambda t} d m_{x, y}(\lambda)$; we recall that this family of measures satisfies the following:

(a) The map $x, y \rightarrow m_{x, y}$ from $H \times H$ into $M_{b}$ is sesquilinear and for all $x \in H, m_{x, x}$ is positive.

(b) $\left|m_{x, y}\right|_{M_{b}} \leqq|x|_{H}|y|_{H}$.

(c) The closure of the union of the supports of $m_{x x}$ for all $x \in H$ is precisely $V$.

Proposition 10. Let $r \in \mathscr{L}$ be subunitary and $R$ its associated unitary representation in $L(H)$. Then,

(i) $\rho_{a, b}$ is the derivative of $m_{a, b}$ for all $a, b \in D$.

(ii) $m_{x, y} \in L^{1}$ and $m_{x, y}\left(V \cap \mathscr{C} V_{D}\right)=0$ for all $x, y \in H$.

(iii) $V_{D}$ is an open dense subset of $V$.

(iv) For each $f \in S(r)$, the restriction of ${ }^{*} T_{f}$ to $E$ is $T_{\bar{f}}$.

(v) For $f, g \in S(r)$ and arbitrary $x, y \in E$,

$$
{ }^{m} T_{f} x, T_{g} y=f \bar{g} m_{x, y} .
$$

Proof. (i) follows from the uniqueness of the fourier transform. From (b) and the fact that $D$ is dense in $H$ we have that $m_{x, y}$ is the limit in $M_{b}$ of a sequence $\left\{m_{a_{n}, b_{n}}\right\}_{n}$ and since $L^{1}$ is a closed subspace of $M_{b}$, we have $m_{x, y} \in L^{1}$. From (i) it is evident that $V_{D} \subseteq V$; if $K$ is any compact set contained in $V \cap \mathscr{C} V_{D}$, we have $m_{a, b}(K)=0$ so that on taking the sup over all $K \subseteq V \cap \mathscr{C} V_{D}, m_{a, b}\left(V \cap \mathscr{C} V_{D}\right)=0$ for all $a, b \in D$. Taking limits in $L^{1}$ gives us $m_{x, y}\left(V \cap \mathscr{C} V_{D}\right)=0$ for all $x, y \in H$. From the fact that $m_{x, x}\left(V \cap \mathscr{C} V_{D}\right)=0$ for all $x \in H$, it follows that $V \cap \mathscr{C} V_{D}$ has a void interior since any nonvoid interior must contain an interior point of the support of $m_{x, x}$ for some $x \in H$. For each $x, y \in E,\left\langle x,{ }^{*} T_{f} y\right\rangle=\left\langle T_{f} x, y\right\rangle$ $=\int\langle r(t) x, y\rangle d m_{f}(t)=\int\left\langle x,{ }^{*} r(t) y\right\rangle d m_{f}(t)=\int\langle x, r(-t) y\rangle d m_{f}(t)$ from which it follows that ${ }^{*} T_{f} y=\int r(-t) y d m_{f}(t)=\int r(t) y d m_{\bar{f}}(t)=T_{\bar{f}} y$, which proves (iv). Again, using (b) and the fact that $D$ is dense in $H$, it is evident that it is sufficient to prove the equality in (v) over $D$; now, for arbitrary $a, b \in D$, 


$$
\int e^{i \lambda t} d m_{T_{f} a, T_{\bar{g}} b}(\lambda)=\left\langle r(t) T_{f} a, T_{g} b\right\rangle=\left\langle T_{\bar{g}} r(t) T_{f} a, b\right\rangle, \text { by (iv). }
$$

Propositions 1 and 2 imply that $\left\langle T_{\bar{g}} r(t) T_{f} a, b\right\rangle=\int e^{i \lambda t} f_{\bar{g}} \rho_{a, b} d \lambda$. Hence $\mid e^{i \lambda t} f \bar{g} \rho_{a, b} d \lambda=\int e^{i \lambda t} d m_{T_{f} a, T_{g} b}(\lambda)$ and with uniqueness of the fourier transform we have $f_{\bar{g}} m_{a, b}=m_{T_{f} a, T_{g} b}$.

We recall that a basic measure for $R[4, \mathrm{p} .112]$ is a positive measure $m$ over $V$ such that for every subset $N$ of $V, m(N)=0$ if and only if $m_{x, x}(N)=0$ for all $x \in H$. Hence, from (ii), we have $m\left(V \cap \mathscr{C} V_{D}\right)=0$.

We are now able to prove an approximation theorem for the weak eigenvectors of subunitary $r \in \mathscr{L}_{1}$.

THEOREM 3. If $r \in \mathscr{L}_{1}$ is subunitary, then for arbitrary $(A, B) \in \mathscr{V}$ and $\lambda \in A, \mathscr{E}_{\lambda}$ is in the uniform closure in $E^{\prime}$ of $\operatorname{Im} p(A, B)$.

Proof. Let $e$ be an arbitrary element of $\mathscr{E}_{\lambda}$; since $E$ is dense in $E^{\prime}$, there exists a sequence $\left\{x_{n}\right\}_{n} \subseteq E$ such that $\lim _{n} x_{n}=e$ in $E^{\prime}$. Hence, using (iv) of Proposition 10, $\lim _{n} T_{\bar{f}} x_{n}=\lim _{n}{ }^{*} T_{f} x_{n}={ }^{*} T_{f} e=f(\lambda) e$, so that for $p(A, B)$, which comes from a real function in $S(r)$, we have $\lim _{n} p(A, B) x_{n}=e$ in $E^{\prime}$.

The following is a somewhat more dramatic version of the same idea.

THeOREM 4. If $r \in \mathscr{L}_{1}$ is subunitary, then for arbitrary $\lambda \in V_{D}$, there exists a sequence $E_{n}$ of closed subspaces of $E$ invariant under the action of $r$ such that $\bigcap_{n} E_{n}=\{0\}$ and for each $n, \mathscr{E}_{\lambda}$ is contained in the uniform closure of $E_{n}$ in $E^{\prime}$.

Proof. Since $\lambda \in V_{D}$, there exists a sequence $\left(A_{n}, B_{n}\right) \in \mathscr{V}$ such that $\bigcap B_{n}=\{\lambda\}$. Define $E_{n}$ as the closure in $E$ of $\operatorname{Im} p\left(A_{n}, B_{n}\right) ; E_{n}$ is evidently invariant under the action of $r$ and Theorem 3 asserts that $\mathscr{E}_{\lambda}$ is contained in the uniform closure of $E_{n}$ in $E^{\prime}$.

Let $z \in \bigcap_{n} E_{n} \cdot m_{z, z}$, by Proposition 10 , is in $L^{1}$ and so is a nonatomic measure. We will now show that the support of $m_{z, z}$ reduces to $\{\lambda\}$ and so it will follow that $m_{z, z}=0$ and consequently $z=0$.

As $z \in E_{n}$, for each $n, z=\lim _{i} x_{i}$ in $E, x_{i} \in \operatorname{Im} p\left(A_{n}, B_{n}\right)$; hence, using (v) of Proposition 10, we conclude that the support of $m_{x_{i}, x_{i}}$, for each $i$ is contained in $B_{n}$, and as $\lim _{i} m_{x_{i}, x_{i}}=m_{z, z}$ in $M_{b}$, we may conclude that the support of $m_{z, z}$ lies in $B_{n}$ for all $n$. From this, it follows that the support of $m_{z, z}$ reduces to $\{\lambda\}$.

As a preliminary to the statement and proof of our main result for subunitary $r \in \mathscr{L}_{1}$, we need a statement of the connection between the multiplicity of $R$ and the eigenfunction expansion for $r \in \mathscr{L}$. We recall that the multiplicity of $R$ may be defined in terms of a direct integral decomposition of $R \oplus \int H_{\lambda} d m(\lambda), m$ a basic measure for $R$, namely, the multiplicity of $R$ is the class of $m$-measurable functions $[\sigma]$ determined by the function $\sigma$ defined by $\sigma(\lambda)=$ the dimension of $H_{\lambda}$ and the relation $\sigma_{1} \in[\sigma]$ if and only if $\sigma_{1}=\sigma$ almost everywhere with respect to $m$. The class $[\sigma]$ does not depend upon the particular direct integral diagonal- 
izing $R$. For an exposition of the theory of direct integrals, we refer the reader to Chapter II of [4].

LEMMA. If $r \in \mathscr{L}$ is subunitary and $R$ is its associated unitary representation in $L(H)$ whose multiplicity $\sigma=1$ a.e. with respect to $m$, then for all $\lambda \in V_{D}$, $\operatorname{dim} \operatorname{Im} T_{\lambda}=1$.

Proof. By virtue of (i) of Proposition 10 and the fact that for any point $\lambda \in V_{D}$ there exists an $a \in D$ such that $\rho_{a, a}$ is strictly positive in a neighborhood of $\lambda$, it follows that the restriction of $m$ to $V_{D}$ is equivalent to the restriction of the lebesgue measure to $V_{D}$. As a consequence of this and (ii) of Proposition $10 \mathrm{~m}$ may be chosen to be the measure over $V$ which is the restriction of lebesgue measure to $V_{D}$ and satisfies $m\left(V \cap \mathscr{C} V_{D}\right)=0$. We now construct a direct integral for $R$.

For each $\lambda \in V_{D}$, define $H_{\lambda}$ to be the nonzero hilbert space obtained from the pre-hilbert space $D$ given the positive hermitian form $a, b \rightarrow \rho_{a, b}(\lambda)$, and for $\lambda \in V \cap \mathscr{C} V_{D}$, define $H_{\lambda}=0$. Hence, by construction, the natural map of $D$ into $H_{\lambda}, a \rightarrow a(\lambda)$ satisfies $[a(\lambda), b(\lambda)]_{H_{\lambda}}=\rho_{a, b}(\lambda)$. Since $\rho_{a, b}$ is integrable, we may form the direct integral $\oplus \int_{V} H_{\lambda} d \lambda$, and it is easy to verify that the map of $D$ into $\oplus \int_{V} H_{\lambda} d \lambda$ defined by $a \rightarrow\{a(\lambda)\}_{\lambda \in V}$ extends to an isometry of $H$ onto $\oplus \int_{V} H_{\lambda} d \lambda$ which diagonalizes $R$. Hence, $\oplus_{V} H_{\lambda} d \lambda$ is a direct integral for $R$, and so $\operatorname{dim} H_{\lambda}=1$ a.e. with respect to the restriction of the lebesgue measure to $V_{D}$; or equivalently, we have $\operatorname{dim} \operatorname{Im} T_{\lambda}=1$ a.e. over $V_{D}$.

We know that $\operatorname{dim} \operatorname{Im} T_{\lambda} \geqq 1$ for all $\lambda \in V_{D}$. Let us assume there exists a $\lambda_{0} \in V_{D}$ such that $\operatorname{dim} T_{\lambda_{0}}>1$. Then, there exists $b_{1}, b_{2} \in D$ such that $\left\{T_{\lambda_{0}} b_{1}, T_{\lambda_{0}} b_{2}\right\}$ is linearly independent. Since $\operatorname{dim} \operatorname{Im} T_{\lambda} \geqq 1$ a.e. over $V_{D}$, there does not exist a neighborhood about $\lambda_{0}$ such that $\left\{T_{\lambda} b_{1}, T_{\lambda} b_{2}\right\}$ is linearly independent for all $\lambda$ in this neighborhood. Hence, there exists a compact neighborhood $V_{0}$ of $\lambda_{0}$ such that $T_{\lambda} b_{1} \neq 0, T_{\lambda} b_{2} \neq 0$ for all $\lambda \in V_{0}$, a sequence $\left\{\lambda_{n}\right\}_{n} \subseteq V_{0}$ such that $\lim _{n} \lambda_{n}=\lambda_{0}$, and a sequence of complex numbers $\left\{k_{n}\right\}_{n}$ whose moduli are bounded above and below, such that

$$
T_{\lambda_{n}} b_{1}=k_{n} T_{\lambda_{n}} b_{2} \text {. }
$$

Now, there exists a subsequence $k_{n_{i}}$ such that $\lim _{i} k_{n_{i}}=k \neq 0$. Therefore, applying $a \in D$ to each side of $\left(^{*}\right)$ and using continuity we have $\left\langle a, T_{\lambda_{0}} b_{1}\right\rangle=\lim _{i}\left\langle a, T_{\lambda_{i}} b_{1}\right\rangle$ $=\lim _{i}\left\langle a, k_{n_{i}} T_{\lambda_{n_{i}}} b_{2}\right\rangle=\left\langle a, k T_{\lambda_{0}} b_{2}\right\rangle$ which implies that $T_{\lambda_{0}} b_{1}=k T_{\lambda_{0}} b_{2}$, which is a contradiction. Hence, $\operatorname{dimIm} T_{\lambda}=1$ for all $\lambda \in V_{D}$.

We may now prove our principal result concerning subunitary $r \in \mathscr{L}_{1}$.

THEOREM 5. Let $r \in \mathscr{L}_{1}$ be subunitary and $R$ its associated unitary representation in $L(H)$ with multiplicity function $\sigma$. If

(a) $\int p_{r}(t) w_{r}(t) d t<+\infty$,

(b) $\sigma=1$ a.e. with respect to a basic measure for $R$, then, for $\lambda \in S \cap V_{D}, \mathscr{E}_{\lambda}$ is one-dimensional and $\mathscr{E}_{\lambda}=\operatorname{Im} T_{\lambda}$. 
Proof. The preceding lemma tells us that the kernel of $T_{\lambda}$, since $\lambda \in V_{D}$, is a closed hyperplane in $D$, which we denote by $X$; since $\mathscr{E}_{\lambda} \neq 0$, to show that $\mathscr{E}_{\lambda}=\operatorname{Im} T_{\lambda}$, it is sufficient to show that $X \subseteq\left\{a:\langle a, e\rangle=0\right.$, for all $\left.e \in \mathscr{E}_{\lambda}\right\}$. Let $a \in X$; since $a, b \rightarrow \rho_{a, b}(\lambda)$ is a positive hermitian form over $D$ so that the CauchySchwartz inequality holds, we have $\rho_{a, b}(\lambda)=0$ for all $b \in D$. Since $\lambda \in V_{D}$, there exists $c \in D$ such that $\rho_{c, c}>0$ over some closed interval $I$ containing $\lambda$ in its interior. Condition (a) again gives us the fact that $\rho_{a, a}$ and $\rho_{a, c}$ are functions in $A(r)$, so that Beurling's theorem asserts that there exists $f_{1} \in A(r)$ such that $f_{1}=1 / \rho_{c, c}$ over $I$. Set $f_{0}=\rho_{a, c} f_{1}$. Since $A(r)$ is regular, there exists $g \in A(r)$ such that $g(\lambda)=1$ and $g$ vanishes outside of $I$. Therefore, $f_{0} g \rho_{c, c}=g \rho_{a, c}$ everywhere, or, in terms of the vector fields in a direct integral diagonalizing $R$, $\{x(\xi)\}_{\xi \in V},\{y(\xi)\}_{\xi \in V}$, and $\{c(\xi)\}_{\xi \in V}$, where $x=T_{f_{0} g} c$ and $y=T_{g} a$, we have

$$
[x(\xi), c(\xi)]_{H}=[y(\xi), c(\xi)]_{H} \text { for all } \xi \in V .
$$

Since $\sigma=1$ a.e. over $V_{D}$ and the vector field $c(\xi)$ does not vanish a.e. over $I$ $\left(^{*}\right)$ implies that $x(\xi)=y(\xi)$ a.e. over $I$, and, as both vector fields vanish outside of $I$, we have $x(\xi)=y(\xi)$ a.e. over $V$ with respect to a basic measure for $R$; from this it follows that $x=y$, or

$$
T_{f_{0 g}} c=T_{g} a .
$$

Now, exactly as in the proof of Theorem 2, $\left(^{* *}\right)$ and Proposition 3 yield, for arbitrary $e \in \mathscr{E}_{\lambda}$, the relation $f_{0}(\lambda) g(\lambda)\langle c, e\rangle=\left\langle T_{f_{0} g} c, e\right\rangle=\left\langle T_{g} a, e\right\rangle=g(\lambda)\langle a, e\rangle$, and the fact that $\rho_{a, c}(\lambda)=0$ implies that $f_{0}(\lambda)=0$, so that $\langle a, e\rangle=0$.

We have the following two corollaries analogous to the first two corollaries from Theorem 2 and whose proofs are exactly the same.

COROLlaRY 1. If $r$ satisfies the conditions of Theorem 5, then for any $\lambda \in V_{D}$ such that $\operatorname{Im} T_{\lambda} \subseteq \mathscr{E}_{\lambda}, \operatorname{Im} T_{\lambda}=\mathscr{E}_{\lambda}$ and both of these spaces are one-dimensional.

COROLlaRY 2. Let $r$ satisfy the conditions of Theorem 5 and assume that $N$ is a linear space dense in $D$ and left invariant by $r(t)$, for all $t$. Then, given any $\lambda \in V_{D}$ for which $\mathscr{N}_{\lambda} \subseteq E^{\prime}$, we must have $\mathscr{N}_{\lambda}=\operatorname{Im} T_{\lambda}=\mathscr{E}_{\lambda}$, and each of these spaces is one-dimensional.

We conclude this section with the following 'concrete' representation theorem for the functional calculus of $r$.

THEOREM 6. If $r$ satisfies the conditions of Theorem 5 and the complement of $S$ in $V_{D}$ is of lebesgue measure zero, then there exist nonzero $e_{\lambda}, f_{\lambda} \in \mathscr{E}_{\lambda}$ for all $\lambda \in V_{D} \cap S$ such that for all $f \in S(r),\left\langle T_{f} x, y\right\rangle=\int_{V_{D^{\cap S}}} f\left\langle x, e_{\lambda}\right\rangle\left\langle\overline{y . f_{\lambda}}\right\rangle d \lambda$, for all $x, y \in E$.

Proof. Since $\operatorname{Im} T_{\lambda}$ is one-dimensional for all $\lambda \in V_{D}$ there exists $e_{\lambda}, f_{\lambda} \in D^{\prime}$ such that $\left\langle a, T_{\lambda} b\right\rangle=\left\langle a, e_{\lambda}\right\rangle\left\langle\overline{b, f_{\lambda}}\right\rangle$ for all $a, b \in D$; as $T_{\lambda} \neq 0$ over $V_{D}, e_{\lambda}$ and $f_{\lambda}$ also cannot vanish over $V_{D}$. Now if $a \in \operatorname{ker} e_{\lambda}$, it follows that 


$$
0=\left\langle a, e_{\lambda}\right\rangle\left\langle\overline{b, f_{\lambda}}\right\rangle=\left\langle\overline{b, e_{\lambda}}\right\rangle\left\langle a, f_{\lambda}\right\rangle,
$$

for all $b \in D$, since $(a, b) \rightarrow\left\langle a, T_{\lambda} b\right\rangle$ is hermitian; so, choosing $b$ such that $\left\langle b, e_{\lambda}\right\rangle$ does not vanish, we have $a \in \operatorname{ker} f_{\lambda}$. Since $\operatorname{ker} f_{\lambda}$ and $\operatorname{ker} e_{\lambda}$ are both closed hyperplanes in $D$, we have $\operatorname{ker} e_{\lambda}=\operatorname{ker} f_{\lambda}$, so that $e_{\lambda}$ and $f_{\lambda}$ must be in the same one-dimensional subspace of $D^{\prime}$. If, in addition, $\lambda \in S$, Theorem 5 tells us $e_{\lambda}$ and $f_{\lambda}$ are in $\mathscr{E}_{\lambda}$.

Given arbitrary $x, y \in E$, we write $\rho_{x, y}$ for the function having the property $d m_{x, y}(\lambda)=\rho_{x, y}(\lambda) d \lambda$. Proposition 10 , (ii) implies that $\rho_{x, y}=0$ a.e. over $V \cap \mathscr{C} V_{D}$ and this fact together with the hypothesis that $\mathscr{C} S \cap V_{D}$ is of lebesgue measure zero means that for all $f \in S(r)$, we may write

$$
\left\langle T_{f} x, y\right\rangle=\int f d m_{x, y}=\int f \rho_{x, y} d \lambda=\int_{S \cap V_{D}} f \rho_{x, y} d \lambda .
$$

Now, we construct sequences $\left\{a_{n}\right\}_{n},\left\{b_{n}\right\}_{n}$ in $D$ converging to $x$ and $y$, respectively, in $E$ such that $\lim _{n} \rho_{a_{n}, b_{n}}=\rho_{x, y}$ exists in $L^{1}$ and, in addition, $\lim _{n} \rho_{a_{n}, b_{n}}(\lambda)=\rho_{x, y}(\lambda)$ a.e. with respect to lebesgue measure. If $\lambda \in S \cap V_{D}$, we have $\lim _{n} \rho_{a_{n}, b_{n}}=$ $\lim _{n}\left\langle a_{n}, e_{\lambda}\right\rangle\left\langle\overline{b_{n}, f_{\lambda}}\right\rangle=\left\langle x, e_{\lambda}\right\rangle\left\langle\overline{y, f_{\lambda}}\right\rangle$ so that $\rho_{x, y}(\lambda)=\left\langle x, e_{\lambda}\right\rangle\left\langle\overline{y, f_{\lambda}}\right\rangle$ a.e. (lebesgue measure) in $S \cap V_{D}(* *)$. The result now follows from $\left(^{*}\right)$ and $\left({ }^{* *}\right)$.

4. An application: the representation of a unitary equivalence. Let $r, r^{\prime} \in \mathscr{R}$ be subunitary, where we will distinguish the various structures associated with $r$ and $r^{\prime}$ by a prime ('). Let $R$ and $R^{\prime}$ denote the associated unitary representations of $r$ and $r^{\prime}$ in $L(H)\left(H\right.$ is independent of $r$ and $\left.r^{\prime}\right)$. We will assume there exists a unitary operator $U \in L(H)$ with adjoint $* U$ such that (i) $U R(t)^{*} U=R^{\prime}(t)$ for all $t$ and (ii) $U$ maps $E$ into $E$. Condition (ii) and the Closed Graph theorem imply that the restriction of $U$ to $E$ is a continuous operator from $E$ into $E$; we can denote, without ambiguity, the adjoint of the restriction of $U$ to $E$ by ${ }^{*} U \in L\left(E^{\prime}\right)$. For these circumstances, we have the following representation theorem for $U$.

THEOREM 7. If $r, r^{\prime} \in \mathscr{R}$ and a unitary operator $U \in L(H)$ satisfy the conditions of the preceding paragraph, and, in addition, $r$ satisfies the conditions of Theorem 6, then, there exists $e_{\lambda}^{\prime} \in \mathscr{E}_{\lambda}^{\prime}, f_{\lambda} \in \mathscr{E}_{\lambda}$ for all $\lambda \in S \cap V_{D}$ such that

$$
\left.\langle U x, y\rangle=\int_{S \cap V_{D}}\left\langle x, e_{\lambda}^{\prime}\right\rangle \overline{\left\langle y, f_{\lambda}\right.}\right\rangle d \lambda \quad \text { for all } x, y \in E .
$$

Proof. Since $U x, y \in E$, for $x, y \in E$, and $r$ satisfies the hypothesis of Theorem 6, we have

$$
\langle U x, y\rangle=\int_{S \cap V_{D}}\left\langle U x, e_{\lambda}\right\rangle\left\langle\overline{y, f_{\lambda}}\right\rangle d \lambda,
$$

where $e_{\lambda}, f_{\lambda} \in \mathscr{E}_{\lambda}$, for all $\lambda \in S \cap V_{D}$. Let $e_{\lambda}^{\prime}={ }^{*} U e_{\lambda}$. In order to prove (*), we have only to show that $e_{\lambda}^{\prime} \in \mathscr{E}_{\lambda}^{\prime}$. For each $x \in E$, we have $\left\langle R^{\prime}(t) x, e_{\lambda}^{\prime}\right\rangle=\left\langle U R^{\prime}(t)^{*} U U x, e_{\lambda}^{\prime}\right\rangle$ $=\left\langle R(t) U x, e_{\lambda}\right\rangle=e^{i \lambda t}\left\langle U x, e_{\lambda}\right\rangle=e^{i \lambda t}\left\langle x, e_{\lambda}^{\prime}\right\rangle$, which proves $e_{\lambda}^{\prime} \in \mathscr{E}_{\lambda}^{\prime}$. 
COROLlaRY. If in addition to the hypotheses of Theorem 7, $U$ maps $E$ onto $E$ and $p_{r}^{\prime} \leqq M p_{r}$ for some constant $M$, then $r^{\prime}$ also satisfies the conditions $o$ Theorem 5 so that $\mathscr{E}_{\lambda}$ and $\mathscr{E}_{\lambda}^{\prime}$ are one-dimensional for all $\lambda \in S \cap V_{D} \cap V_{D}^{\prime}$. Furthermore,

$$
\left.\langle U x, y\rangle=\int_{S \cap V_{D} \cap V_{D}^{\prime}}\left\langle x, e_{\lambda}{ }^{\prime}\right\rangle \overline{\left\langle y, f_{\lambda}\right.}\right\rangle d \lambda, \quad x, y \in E .
$$

Proof. $U$, by the Closed Graph theorem, is a topological isomorphism of $E$ onto $E$, so that $U^{-1}={ }^{*} U$ over $E$ and, $S=S^{\prime}$. Also,

$$
\left|r^{\prime}(t)\right|_{L(E)} \leqq\left.\left.|U|_{L(E)}\right|^{*} U\right|_{L(E)}|r(t)|_{L(E)}
$$

so that $r^{\prime} \in \mathscr{L}_{1}$ and $\int p_{r}^{\prime} w_{r}^{\prime} d t<+\infty$, which proves (a) of Theorem 5. Since unitary equivalence preserves in an obvious way the spectrum, the class of equivalent basic measures, and the multiplicity function, we evidently have condition (b) of Theorem 5 also satisfied for $\boldsymbol{r}^{\prime}$. Hence, $\mathscr{E}_{\lambda}$ and $\mathscr{E}_{\lambda}^{\prime}$ are one-dimensional for all $\lambda \in S \cap V_{D} \cap V_{D}^{\prime}$.

Let $m$ denote a basic measure for $R$ and $R^{\prime}$; since the complement of $V_{D} \cap S$ and $V_{D}^{\prime} \cap S$ in $V_{D}$ and $V_{D}^{\prime}$, respectively, is of lebesgue measure zero and, over $V_{D}$ and $V_{D}^{\prime}, m$ is equivalent to lebesgue measure, we have, using (ii) of Proposition $10, m\left(V \cap \mathscr{C}\left(V_{D} \cap S\right)\right)=m\left(V \cap \mathscr{C}\left(V_{D}^{\prime} \cap S\right)\right)=0$. Therefore, $m\left(V \cap \mathscr{C}\left(V_{D} \cap V_{D}^{\prime} \cap S\right)\right)$ $=0$, so that we have $\langle U x, y\rangle=\int d m_{U x, y}=\int_{S \cap V_{D} \cap V_{D}^{\prime}} d m_{U x, y}$ $=\int_{S \cap V_{D^{\cap}} V_{D}^{\prime}}\left\langle U x, e_{\lambda}\right\rangle\left\langle\overline{\left\langle y, f_{\lambda}\right.}\right\rangle d \lambda$, using Theorem 6 for $r$. Now, in the proof of Theorem 7, we showed that $\left\langle U x, e_{\lambda}\right\rangle=\left\langle x, e_{\lambda}^{\prime}\right\rangle$, where $e_{\lambda}^{\prime} \in \mathscr{E}_{\lambda}^{\prime}$ for all $\lambda \in S$, and this establishes $\left({ }^{* *}\right)$.

5. An example ( $\left.{ }^{2}\right)$. We will give an example of an invariant subspace where our results show that the functional calculus may be explicitly constructed from the weak eigenvectors over this space. For the group of operators in $L^{2}\left(R_{3}\right)$ whose infinitesimal generator is $-i \Delta$ where $\Delta$ is the laplacian, we will show that every cyclic subspace generated by a function $x_{0} \in L^{2}\left(R_{3}\right)$ with compact support has such an invariant subspace.

We consider a strongly continuous one-parameter group of unitary operators $R$ in $L(H)$ where $H$ is a cyclic space for $R$ generated by $x_{0} \in H$. Denote by $A$ the usual banach algebra of fourier transforms of $L^{1}$-functions and, by $W$, the subalgebra of $A$ consisting of all twice differentiable functions such that $f, f^{\prime}$, and $f^{\prime \prime}$ are all in $A$. Let $G$ denote the interior of the spectrum $V$ of the infinitesimal generator of $R$ ( $G$ is also the interior of the support of the measure $m_{x_{0}, x_{0}}$ ). We impose the following condition on $x_{0}$ :

$$
m_{x_{0}, x_{0}} \text { is locally in } W \text { over } G \text { and } m_{x_{0}, x_{0}}(V \cap \mathscr{C} G)=0 \text {. }
$$

We construct $E$. Define $\Phi$ to be the subspace of $H$ consisting of all $T_{f} x_{0}$, where $f \in W$ and $f$ has compact support in $G$.

(2) Added March, 231963. 
$\Phi$ is invariant under the action of $R$ and we have $\left\langle R(\quad) x, x_{0}\right\rangle \in L^{1}$ for all $x \in \Phi$. Introduce over $\Phi$ the norm sup $\left\{\int\left|\left\langle R(t) x, x_{0}\right\rangle\right| d t,|x|_{H}\right\}$ and define $E$ to be the completion of $\Phi$ with respect to this norm. It may be shown that $E$ may be regarded as a subset of $H$ and the natural map of $E$ into $H$ is continuous. Furthermore, $E$ is invariant under $R$, and if $r$ denotes the representation obtained by restricting $R(t)$ to $E$, we have that $r(t)$ is an isometry for each $t$; using the fact that translation is continuous in $L^{1}$, it follows that $r$ is a strongly continuous one-parameter group of operators in $L(E)$.

Proposition 11. If $\left(^{*}\right)$ holds, then there exists a separable banach space $D \subseteq E$, continuously and densely imbedded in $E$ for which

$$
\sup _{|a|_{D},|b|_{D} \leqq 1}|\langle r(t) a, b\rangle|
$$

is in $L^{1}$ and the union of the interiors of the supports of $m_{a, a}$ for $a \in D$ is precisely $G$.

This construction of $D$ has been relegated to the Appendix.

Regarding $D$ and $E$ imbedded in $D^{\prime}$ via the transpose of the natural map of $D$ and $E$ in $H$, we note that our general requirements are satisfied and we have:

Proposition 12. $r$ satisfies the hypotheses of Theorem 6 with $V_{D}=G$ and $S \subseteq G$ (i.e., for each $\lambda \in G, \mathscr{E}_{\lambda} \neq 0$ ).

Proof. All except the fact that $S \subseteq G$ is immediate from our construction. To prove $S \subseteq G$, we note that the map

$$
x \rightarrow \rho_{x, x_{0}}(\lambda) \quad\left(\rho_{x, x_{0}}=m_{x, x_{0}}=\left[\left\langle R(\quad) x, x_{0}\right\rangle\right]^{\wedge}\right)
$$

for $\lambda \in G$ is $a$ continuous nonzero linear functional over $E$ and so is represented by a nonzero $e_{\lambda} \in E^{\prime}$; as $\rho_{r(t) x, x_{0}}(\lambda)=e^{i \lambda t} \rho_{x, x_{0}}(\lambda)$, we have $e_{\lambda} \in \mathscr{E}_{\lambda}$.

Theorem 6 now informs us that for all $\lambda \in G, \mathscr{E}_{\lambda}$ is one-dimensional and there exists nonzero $e_{\lambda}, f_{\lambda} \in \mathscr{E}_{\lambda}$ such that

$$
\left.\langle R(t) x, y\rangle=\langle r(t) x, y\rangle=\int_{G} e^{i \lambda t}\left\langle x, e_{\lambda}\right\rangle \overline{\left\langle y, f_{\lambda}\right.}\right\rangle d \lambda, \quad x, y \in E .
$$

Furthermore, $E$ is invariant under an operator $T_{f}$ in the spectral calculus for $R$ corresponding to a function $f \in A$. We emphasize here the fact that this result and Proposition 12 are independent of $D$.

For the group of operators $R(t) \in L^{2}\left(R_{3}\right)$ given by the solution of the Schroedinger equation

$$
\frac{1}{i} \frac{\partial}{\partial t}=-\Delta
$$

we know that a basic measure for $R$ is equivalent to lebesgue measure over the interior of the spectrum of $R$, the interval $[0, \infty)$. Also, 


$$
\rho_{x_{0}, x_{0}}(\lambda)=\frac{1}{4 \pi^{2}} \int_{R_{3} \times R_{3}} \frac{\sin |\xi-\eta| \sqrt{ } \lambda}{|\xi-\eta|} x_{0}(\xi) x_{0} \overline{(\eta)} d \xi d \eta \text { a.e. } \lambda>0
$$

$\left[9\right.$, p.132]. If $x_{0}$ has its support in a sphere of radius $k$, then

$$
\rho_{x_{0}, x_{0}}(\lambda)=\sum_{n=0}^{\infty} \frac{(-1)^{n}\left(\lambda^{1 / 2}\right)^{2 n+1}}{(2 n+1) !} \alpha_{n} \quad \text { a.e., } \lambda>0,
$$

where $\alpha_{n}=\left(1 / 4 \pi^{2}\right) \int_{R_{3} \times R_{3}}|\xi-\eta|^{2 n} x_{0}(\xi) \overline{x_{0}(\eta)} d \xi d \eta$ and $\left|\alpha_{n}\right| \leqq(2 k)^{2 n}\left|x_{0}\right|_{L^{\prime}}^{2} / 4 \pi^{2}$.

It follows that $\rho_{x_{0}, x_{0}}(\lambda)$ for $\lambda>0$ is the restriction of a function analytic in a disk about $\lambda^{\prime}>\lambda$ not containing 0 ; hence, $\rho_{x_{0}, x_{0}}$ is locally in $W$ over $G \subseteq(0, \infty)$. As the zeros of $\rho_{x_{0}, x_{0}}$ are at most countable in $[0, \infty)$ and $m_{x_{0}, x_{0}}$ is absolutely continuous, we have that $m_{x_{0}, x_{0}}(V \cap \mathscr{C} G)=0(V=[0, \infty))$. In short, we have shown that $\left(^{*}\right)$ is satisfied for $R$ generated by $-i \nabla$ in $L^{2}\left(R_{3}\right)$ (with its maximal domain) and $x_{0}$ with compact support. A similar result holds for the laplacian in $L^{2}\left(R_{n}\right)$ for arbitrary $n \geqq 2$.

\section{Appendix to $\$ 5$.}

Proof of Proposition 11. First, we observe that every $x \in \Phi$ may be written uniquely in the form $T_{f} x_{0}$, where $f \in W$ with support in $G$; furthermore, denoting $\rho_{x_{0}, x_{0}}^{1 / 2}$ by $\theta$, we have $\theta f \in W$, which implies $\left|[\theta f]^{\wedge}(t)\right|=O\left(1+t^{2}\right)^{-1}$.

Now, define $Q$ as the set of $x \in \Phi$, such that

(i) $|x|_{E} \leqq 1$.

(ii) $\left|[\theta f]^{\wedge}(t)\right| \leqq 1 /\left(1+t^{2}\right), T_{f} x_{0}=x$.

$Q$ is convex, so that the closure $\bar{Q}$ of $Q$ in $E$ is bounded, convex, and circled; consequently, there is a unique banach space $D_{1}$ such that $\bar{Q}$ is precisely the unit ball of $D_{1} . D_{1}$ is not, a priori, separable, but we can construct a smaller subspace which is separable. In order to do this, we need the following:

LEMMA. The topology induced by $E$ over $\Phi$ is separable.

Proof. Since $\Phi$ is given the least upper bound of two topologies one of which is known to be separable, we need only show $\Phi$ is separable with respect to the topology induced by the norm $\int\left|\left\langle R(t) x, x_{0}\right\rangle\right| d t=|x|_{1}$. $A$ is separable, so that the subspace of all $f \theta^{2}$ where $f$ is in $W$ and $f$ has support in $G$, is also separable; call this subspace of $A, I$. The map of $f \theta^{2}$ onto $T_{f} x_{0}$ is an isometry of $\Phi$ with the norm ||$_{1}$ onto $I$; hence $\Phi$ with topology induced by the ncrm ||$_{1}$ is separable. This completes the proof of the lemma.

$\Phi$ is dense in $E$ so that by the lemma, there exists a countable set $\Phi_{c}$ total in $E$ where $\Phi_{c} \subseteq \Phi$. We may also require that the union of the interiors of the supports of $m_{x, x}$ for $x \in \Phi_{c}$ be precisely $G$. (by adding elements of the form $T_{f_{i}} x_{0}=x_{i}$, where the union of the supports of $f_{i}$ is $G, x_{i} \in \Phi$ ).

Now, define $D$ to be the closure in $D_{1}$ of the linear span of $\Phi_{c}$; in consequence of this, $D$ is separable, and $V_{D}=G$. 
We now estimate $\langle R(t) a, b\rangle$ for $a, b$ in the unit ball of $D$; as $Q \cap D$ is dense in the unit ball of $D$, we need only estimate $\langle R(t) a, b\rangle$ for $a, b \in Q$. As $a=T_{f} x_{0}$, $b=T_{g} x_{0}$, we have $|\langle R(t) a, b\rangle|=\left|\int e^{i \lambda t} f \bar{g} \theta^{2} d \lambda\right|=|[f \theta] *[\bar{g} \theta](t)|$ and the latter convolution is dominated by the convolution of $1 /\left(1+t^{2}\right)$ with itself, which is in $L^{1}$. This completes the proof.

\section{BIBLIOGRAPHY}

1. A Beurling, Sur les intégrales de Fourier absolument convergentes et leur applications à une transformation fonctionnelle, Proc. 9th Scandinavian Mathematical Congress, 1938, Helsinki, 1939.

2. - Quasi-analyticity and general distributions, Lecture Notes, Conference in Functional Analysis, Stanford University, Stanford, Calif., 1961.

3. F. Browder, Eigenfunction expansions for non-symmetric partial differential operators. I, II, and III, Amer. J. Math. 80 (1958), 365-381; ibid. 81 (1959), 1-22, 715-734.

4. J. Dixmier, Les algebres d'opérateurs dans l'espace hilbertien, Gauthier-Villars, Paris, 1957.

5. I. M. Gel'fand and G. E. Šilov, Quelques applications de la théorie des fonctions généralisées, J. Math. Pures Appl. 35 (1956), 383-413.

6. E. Hille and R. S. Phillips, Functional analysis and semi-groups, Amer. Math. Soc. Colloq. Publ. Vol. 31, Amer. Math. Soc., Providence, R. I., 1957.

7. L. H. Loomis, Abstract harmonic analysis, Van Nostrand, New York, 1953.

8. R. S. Phillips, Spectral theory for semi-groups of operators, Trans. Amer. Math. Soc. 71 (1951), 393-415.

9. E. C. Titchmarsh, Eigen-function expansions associated with second-order differential equations. II, Clarendon, Oxford, 1958.

10. H. Weyl, Über gewöhnliche Differentialgleichungen mit Singuläritaten und die zugehörigen Entwicklungen willkürlichen Funktionen, Math. Ann. 68 (1910), 220-269.

DUKe UNIVERSITY,

Durham, North Carolina 